\title{
Cavernous Haemangioma of the Orbit
}

\author{
B. LEATHERBARROW, J. L. NOBLE, I. C. LLOYD \\ Manchester
}

\begin{abstract}
Summary
Cavernous haemangioma is considered to be the most common primary orbital tumour. We present 3 cases of cavernous haemangioma of the orbit managed by medial orbitotomy combined with medial orbital decompression. This approach was performed for lesions occurring medial to the optic nerve in an attempt to reduce the surgical morbidity reported following lateral orbitotomy or transcranial orbitotomy.
\end{abstract}

Cavernous haemangioma, often cited as the most common primary orbital tumour, occurs infrequently. ${ }^{1,2}$ As a cause of unilateral proptosis it is outnumbered by primary congenital varices in a ratio of $3: 1 .^{2}$ Cavernous haemangioma is an acquired benign vascular tumour which occurs in adults and exhibits a slow growth pattern. Histologically it is composed of large, dilated vascular channels lined by flattened endothelial cells, and possesses a well-defined fibrous pseudo-capsule. The capsule enables excision of the entire lesion without fragmentation, following which recurrence does not usually occur. Malignant transformation has never been reported in this tumour.

Despite its histologically benign nature, the frequent location within the retrobulbar muscle cone may compress the optic nerve resulting in visual loss. Nevertheless, the natural history of orbital cavernous haemangioma has been shown to be quite variable. Many patients may have manifestations of tumour growth for more than 10 years without developing non-correctable visual loss, whereas others may have visual loss that is not optically correctable within a few months of onset of symptoms. ${ }^{4}$

Wright $^{2}$ feels that cavernous haemangiomas should be excised as soon as the diagnosis can be established. Such an approach is based on the observation that progressive expansion of the lesion eventually leads to optic nerve compression, extreme proptosis with the risk of corneal exposure, and greater difficulty in surgical removal.

Harris and Jakobiec ${ }^{4}$ showed, however, that surgery performed on patients who had a tumour located deep in the orbit causing choroidal folds, disc swelling, envelopment of the optic nerve, periosteum, or muscles, had a greater risk of resulting in a post-operative worsening of visual acuity. Tumours in this location are usually approached by a lateral orbitotomy or a modified Kronlein resection of the lateral orbital wall; occasionally an approach by the transcranial route is necessary.

The purpose of this paper is to report three cases of intraconal cavernous haemangiomas managed by a medial orbitotomy combined with medial orbital decompression to minimise surgical morbidity.

\section{Materials and Methods}

During an 8 year period ending May 1988, three patients with orbital intraconal cavernous haemangioma presented to the Royal Eye Hospital, Manchester. In each case a pre-operative diagnosis of orbital cavernous haemangioma lying medial to the optic nerve was made, and the patient subjected to a 
medial orbitotomy combined with medial orbital decompression to approach the tumour.

The procedure was carried out under general anaesthesia with a cuffed endotracheal tube. A high nasal skin incision was made and after reflecting the medial canthal tendon, periosteum and lacrimal sac laterally, the medial wall of the orbit was resected (Figs, 1,2). The resection included a portion of the anterior lacrimal crest, the orbital plate of the ethmoid, together with the bony septa and mucosa of the ethmoid sinuses. The resection was carried superiorly to the frontoethmoidal suture, and posteriorly to the orbital apex. The periorbita was then incised and the tumour exposed.

The tumour specimens were analysed histologically using formalin fixation, paraffin embedding and haematoxylin and eosin, and Masson's trichrome staining.

\section{Clinical records}

Case 1

A 41 year old male presented with an 8 week history of gradual deterioration of vision in his left eye, accompanied by retrobulbar pain. He gave no history of proptosis or diplopia but had been aware of some prominence of the left eye for some years. There was no significant past medical or associated family history.

Examination showed a $5 \mathrm{~mm}$ axial, non-pulsatile, proptosis of the left eye (Fig. 3). There were no palpable orbital masses, no bruits and no change in the proptosis with a Valsalva manoeuvre. The best corrected visual acuity in the left eye was 6/9. There was a left relative afferent pupillary defect. Visual field examination revealed

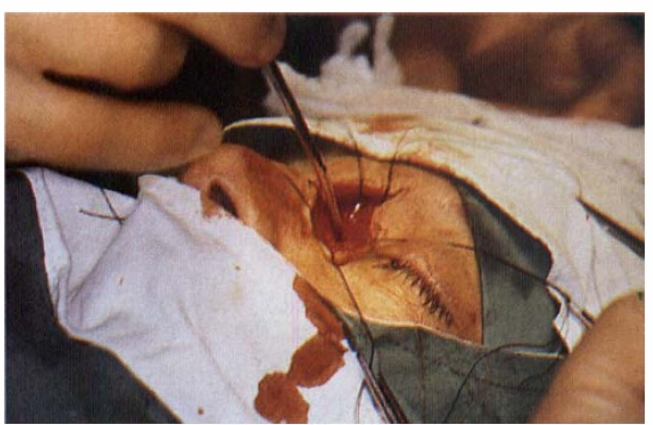

Fig. 1. The surgical incision with reflection of medial canthal tendon and lacrimal sac temporally. a left relative central scotoma. Extraocular muscle movements were normal. Fundoscopy revealed unilateral disc oedema and peripapillary choroidal folds (Fig. 4). General physical examination revealed no abnormalities. $\mathrm{He}$ was clinically euthyroid and thyroid function tests were normal. Plain X-ray examinations of the orbits and chest were normal. Standardised A-scan ultrasonography showed the lesion to have a well-outlined border with high internal reflectivity and medium

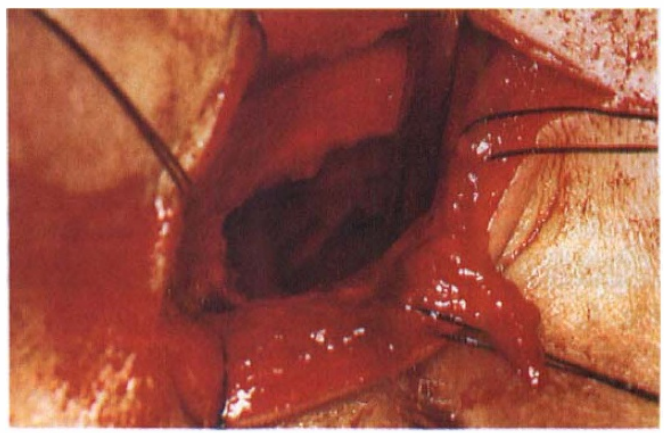

Fig. 2. Medial orbital wall decompression.

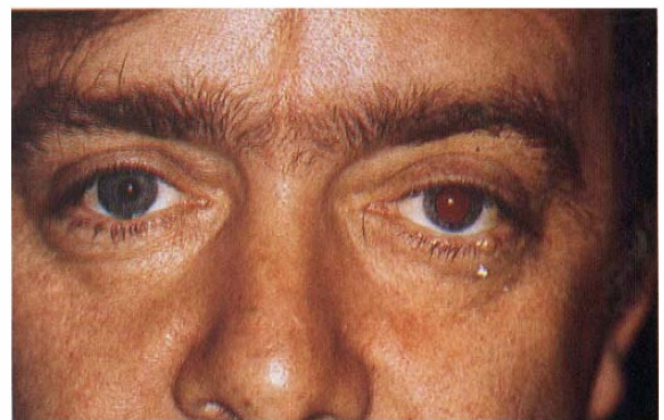

Fig. 3. Case 1: A 41 year old male with left proptosis.

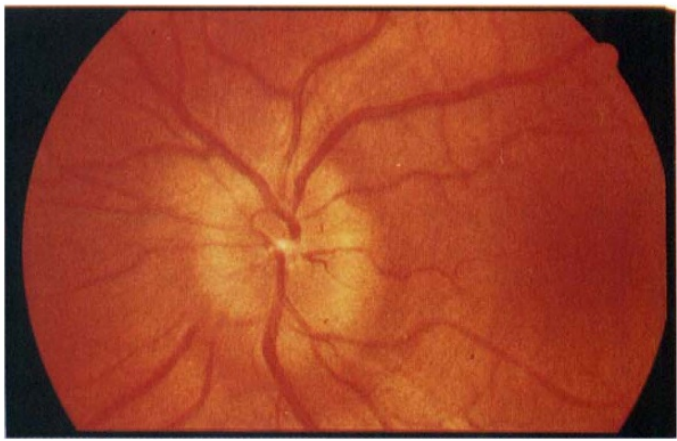

Fig. 4. Case 1: Disc oedema with peripapillary choroidal folds. 
sound attenuation (Fig. 5). B-scan ultrasonography showed a well-circumscribed retrobulbar intraconal lesion with a rounded regular outline and moderate sound transmission (Fig. 6). Computerised axial tomographic (CAT) scans were

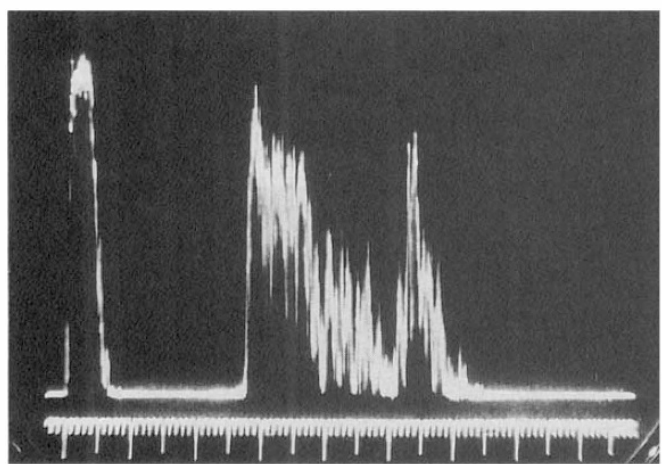

Fig. 5. Case 1: A-mode ultrasonogram of cavernous haemangioma demonstrating moderate internal reflectivity. taken with plain and contrast films. A uniformly enhancing, regular, well-circumscribed, retrobulbar intraconal mass in the left orbit was revealed

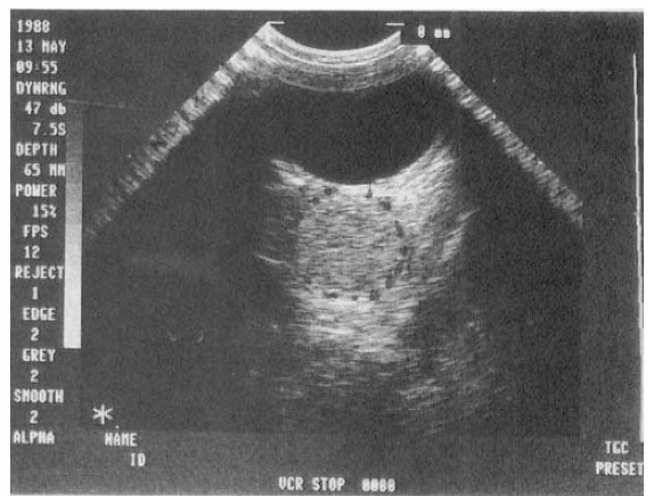

Fig. 6. Case 1: B-mode ultrasonogram of cavernous haemangioma demonstrating welldefined border echoes with good sound transmission.

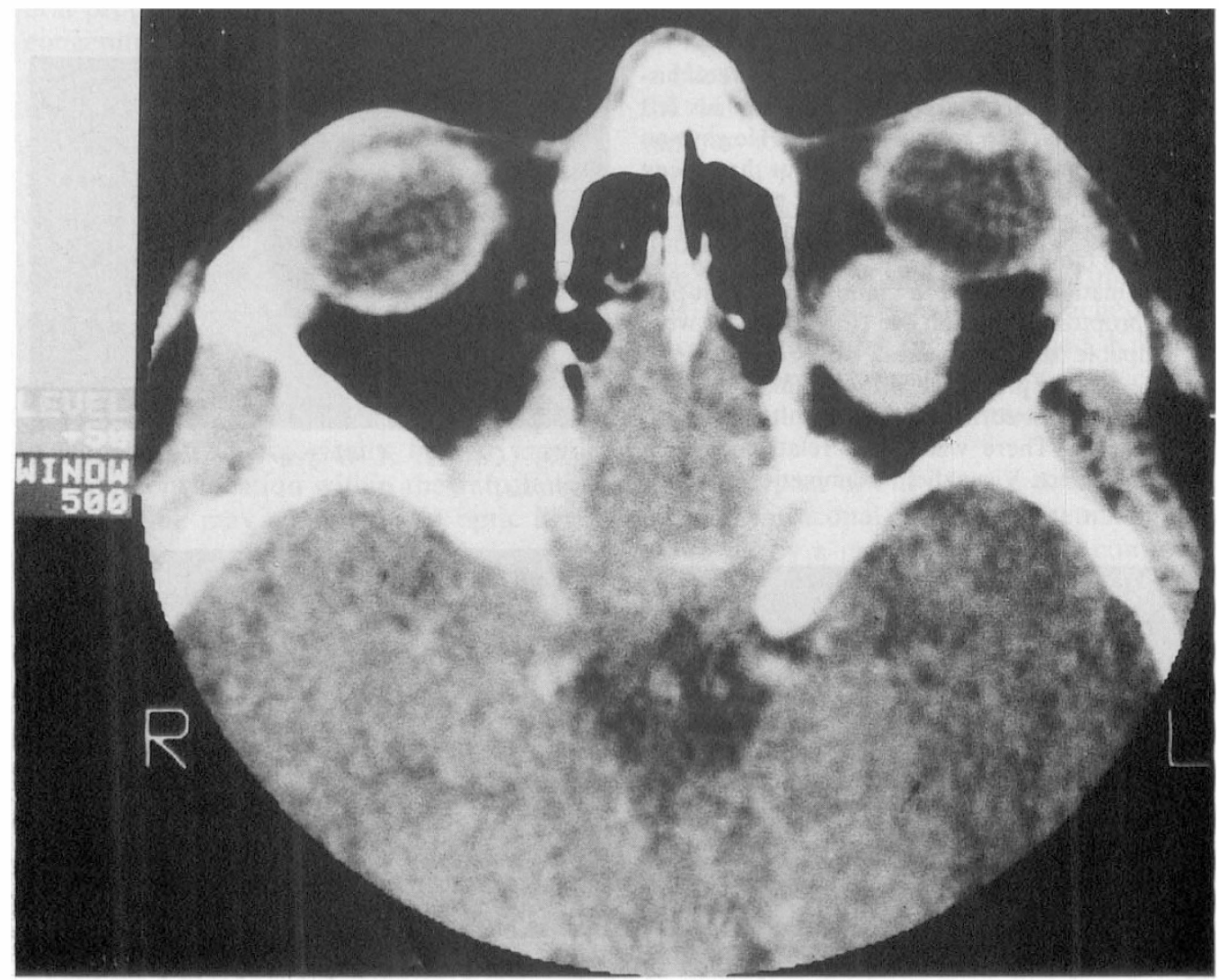

Fig. 7. Case 1: Preoperative CAT scan demon-strating orbital cavernous haemangioma and proptosis. 
(Fig. 7). Angiography and venography were not performed. A preoperative diagnosis of orbital cavernous haemangioma was made.

Two weeks after presentation the patient underwent a left medial orbitotomy and medial orbital decompression as described above. The tumour was easily dissected free from the surrounding structures. During the course of the dissection it prolapsed anteriorly into the supero-temporal fornix whence it was removed as an intact encapsu-

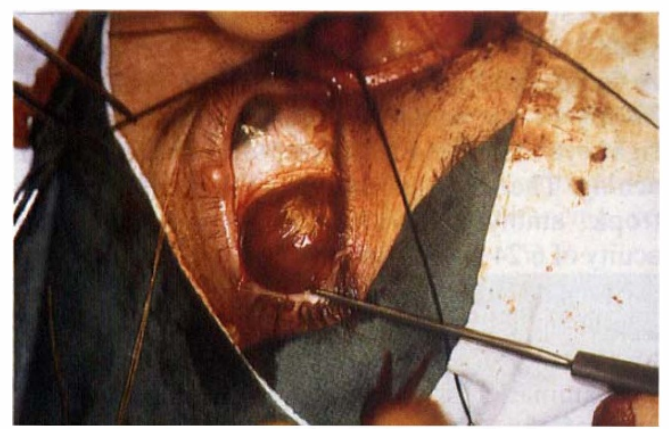

Fig. 8. Case 1: Presentation and removal of cavernous haemangioma.

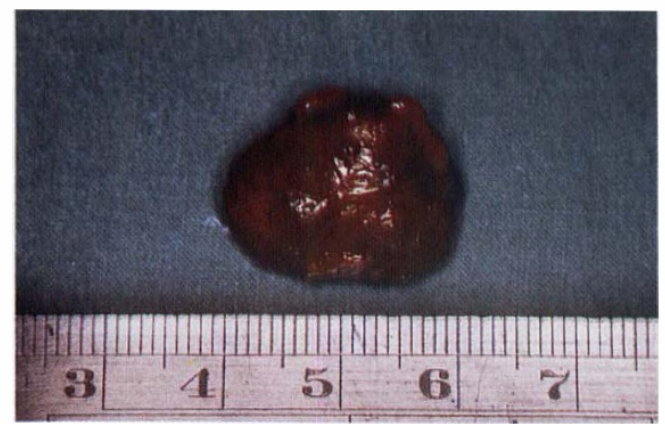

Fig. 9. Case 1: Encapsulated tumour removed completely.

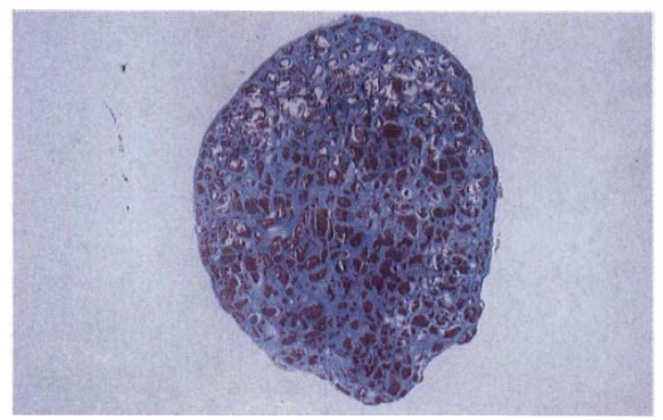

nig. 10. Case 1: Histopathologic appearance of excised tumour. lated firm tumour (Figs. 8,9). It did not have a vascular pedicle. Histopathological analysis showed the tumour to be a benign cavernous haemangioma (Fig. 10).

His postoperative appearance at day one is shown (Fig. 11). At one month follow-up the bestcorrected visual acuity had improved to $6 / 6$. The visual field was normal. The proptosis had completely resolved and the surgical scar was very satisfactory (Fig. 12). At 3 month follow-up there were no problems.

\section{Case 2}

A 53 year old male presented with a 6 month history of diplopia on extreme laevoversion. He did not complain of any visual loss, pain or proptosis. He had no known illnesses and no relevant family history.

Examination showed a $2 \mathrm{~mm}$ axial, non-pulsatile proptosis of the left eye. There were no palpable orbital masses, no bruits and no change in the proptosis with a Valsalva manoeuvre. His unaided visual acuity was $6 / 6$. The visual fields were full and the pupil reactions were normal. Abduction of the left eye was restricted, with horizontal diplopia in extreme laevoversion. Fundoscopy was normal.

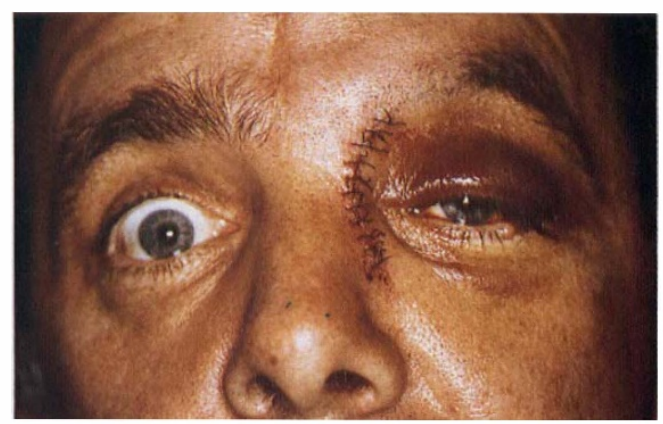

Fig. 11. Case 1: Postoperative appearance Day 1.

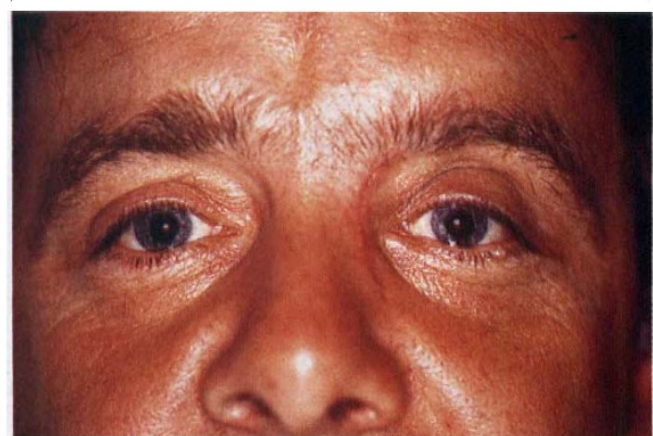

Fig. 12. Case 1: Postoperative appearance Day 28 . 
General physical examination revealed no abnormalities. He was clinically euthyroid and thyroid function tests were normal.

Plain X-ray examinations of the orbits and chest were normal. Standardised A-scan ultrasonography showed the lesion to have a well-outlined border with high internal reflectivity and medium sound attenuation. B-scan ultrasonography showed a well-circumscribed, retrobulbar intraconal mass with moderate sound transmission. CAT scans showed a large, well-defined intraconal mass, which enhanced with contrast, displacing the optic nerve laterally in the left orbit (Figs. $13,14)$. Angiography and venography were not performed. A preoperative diagnosis of orbital cavernous haemangioma was made.

One week after presentation the patient underwent a left orbitotomy and medial orbital decompression as described above. A large orbital mass was found extending postero-medially to the orbital apex. It was found to be enveloping the optic nerve. Careful dissection of the tumour from the optic nerve was considered to be too dangerous. The mass was biopsied.
Histopathological analysis showed the tumour to be a benign cavernous haemangioma.

There were no postoperative complications. At 2 year follow-up the unaided visual acuity was $6 / 6$ and the visual fields were full. There was no proptosis and the diplopia, though still present in extreme laevoversion, was improved. CAT scans performed postoperatively show the medial decompression of the left orbit into which the tumour partially herniates adopting a more pearshaped configuration (Figs. 15, 16).

\section{Case 3}

A 56 year old female presented with an 8 month history of a gradually increasing proptosis of the right eye associated with symptoms of retrobulbar aching. There was a past history of right anisometropic amblyopia with a best-corrected visual acuity of 6/24 in the right eye.

She gave no history of diplopia. There was no significant past medical or associated family history.

Examination showed a $3 \mathrm{~mm}$ non-pulsatile proptosis of the right eye, with the globe displaced

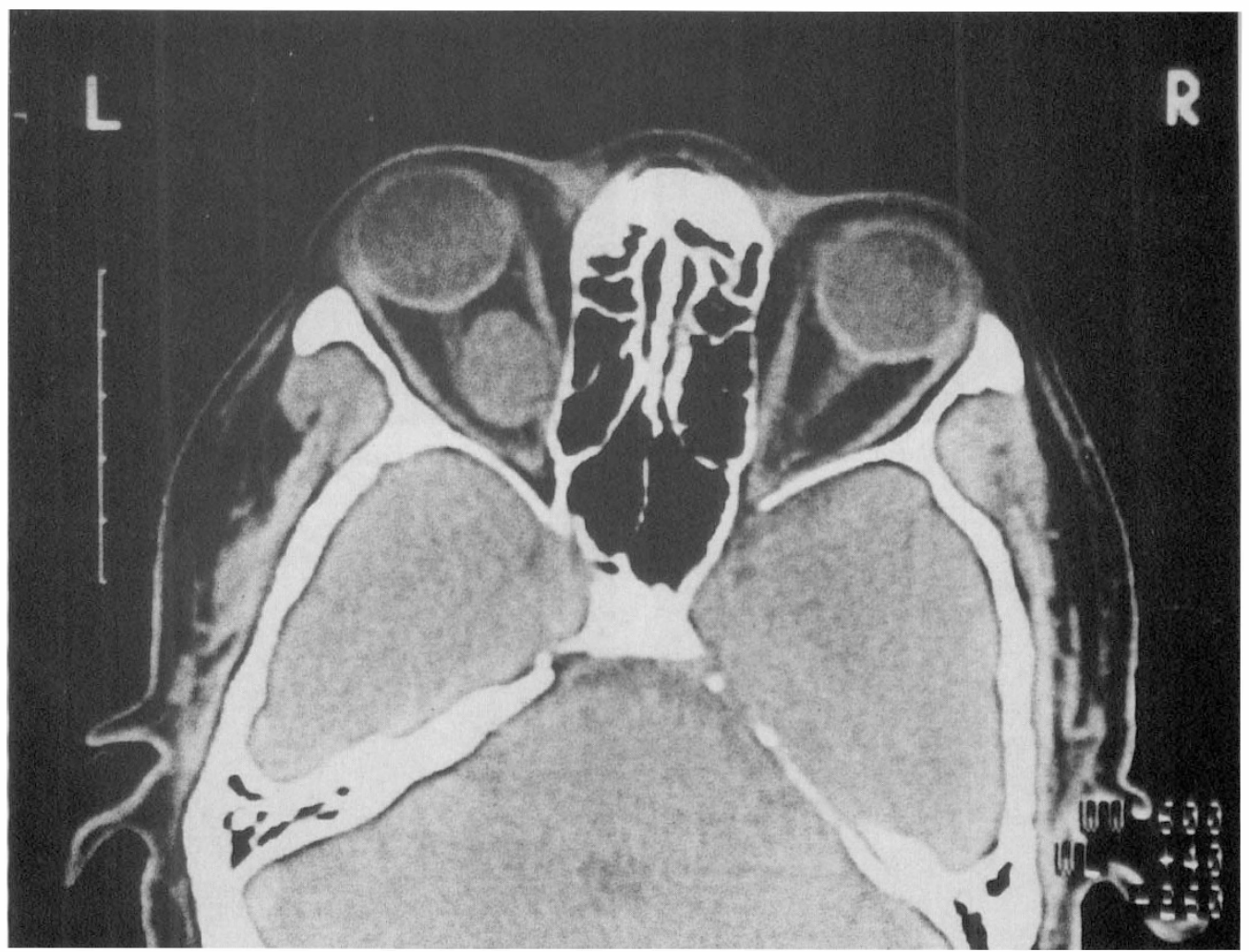

Fig. 13. Case 2: Preoperative CAT scan demon-strating orbital cavernous hemangioma and proptosis. 
inferiorly by $2 \mathrm{~mm}$ and temporally by $5 \mathrm{~mm}$. There were no palpable orbital masses, no bruits and no change in the proptosis with a Valsalva manoeuvre. Her best-corrected visual acuity in the right eye was $6 / 24$. The visual fields were full and the pupil reactions were normal. Extraocular muscle movements were normal. Fundoscopy was normal. General physical examination was normal. She was clinically euthyroid and thyroid function tests were normal. Plain X-ray examinations of the orbits and chest were normal. Standardised Ascan ultrasonography showed the lesion to have a well-outlined border with high internal reflectivity and medium sound attenuation (Fig. 17). B-scan ultrasonography showed a well-circumscribed retrobulbar intraconal lesion with a rounded regular outline and moderate sound transmission. CAT scans showed a uniformly enhancing, regu- lar, well-circumscribed retrobulbar intraconal mass in the right orbit (Fig. 18). A right carotid angiogram and an orbital venogram were performed but these investigations added no further helpful information. A preoperative diagnosis of orbital cavernous haemangioma was made.

Two weeks after presentation the lesion was biopsied via a medial conjunctival approach. Histopathological analysis confirmed the tumour to be a benign orbital cavernous haemangioma. Postoperatively the patient had slight restriction of adduction in the right eye with diplopia on extreme dextroversion, which persisted. Therefore, two years after initial presentation, at the patient's request, she underwent a right medial orbitotomy and medial orbital decompression as described above.

The tumour was carefully dissected from the

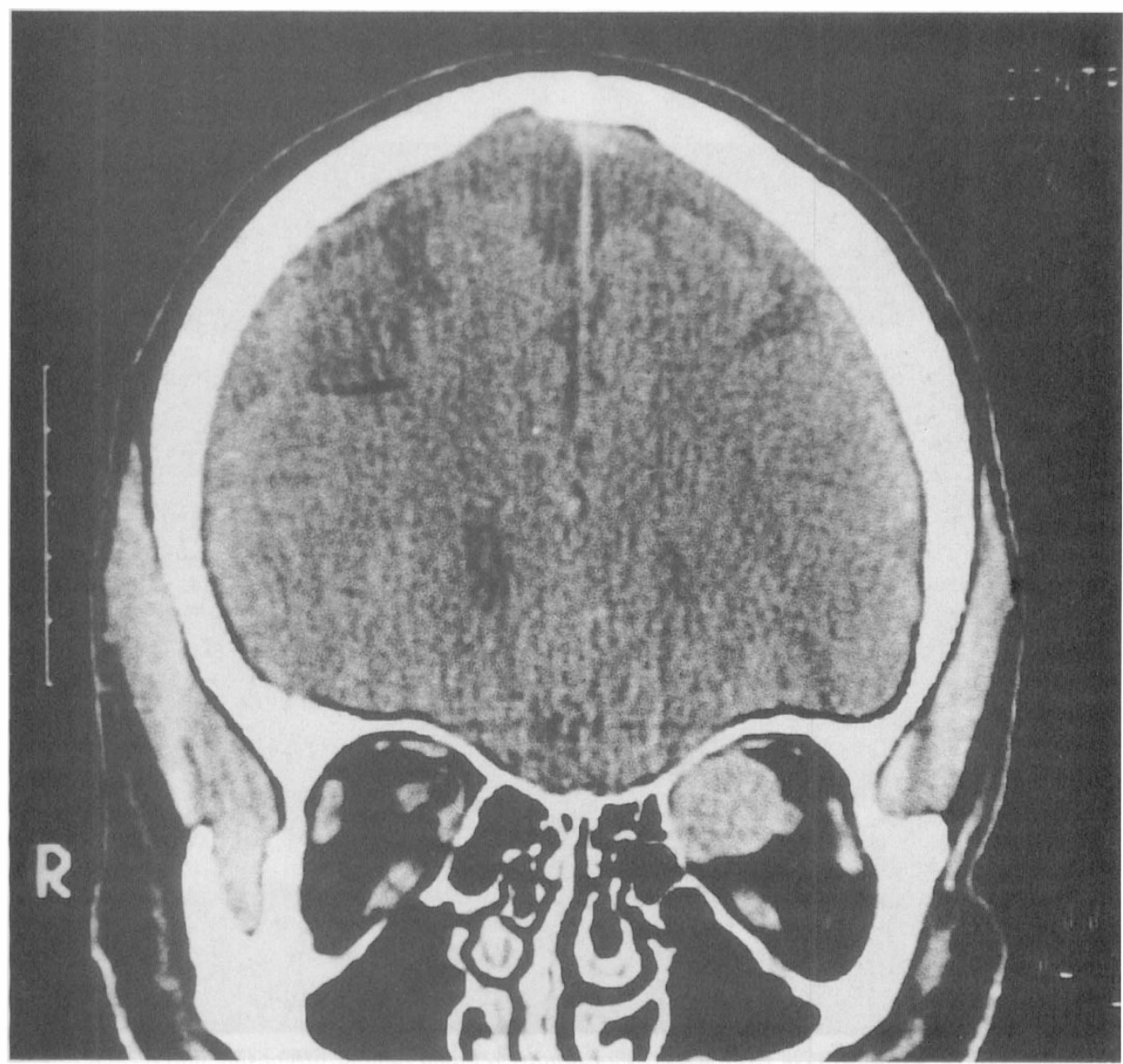

Fig. 14. Case 2: Preoperative CAT scan: Coronal view. 
optic nerve to which it was adherent. It was removed intact and did not have a vascular pedicle. There were no postoperative complications. At 6 year follow-ups the best-corrected visual acuity was $6 / 24$. The visual field was full. There was no proptosis. The extraocular muscle movements and diplopia were unchanged.

\section{Discussion}

The symptoms of cavernous haemangioma of the orbit characteristically develop gradually over a period of months to years. The presenting symptoms vary from patient to patient as shown by our group of three cases. The symptoms typically are those of slowly progressive proptosis, with or without extraocular motility problems and visual disturbance. This is a disorder which occurs most frequently in middle-aged adults with a female predominance. ${ }^{4}$
The following investigations are mandatory in the preoperative evaluation of these patients.:

(1) CAT scan examination using plain and contrast enhancing films in all cases. This is the single most useful test in evaluating these patients. All cavernous haemangiomas show contrast enhancement after intravenous infusion of contrast. ${ }^{5}$ The typical CT findings are a rounded, well-circumscribed appearance with no association inflammation or infiltration of adjacent structures. Coronal CT is of value as it relates the optic nerve to the tumour for operative approach. CT is also sensitive in detecting apical extension. ${ }^{5}$

(2) Standardised A-scan and B-scan ultrasonography. The ultrasonographic characteristics of orbital cavernous haemangioma have been described by Coleman. ${ }^{6}$ Arteriography and venography have no role

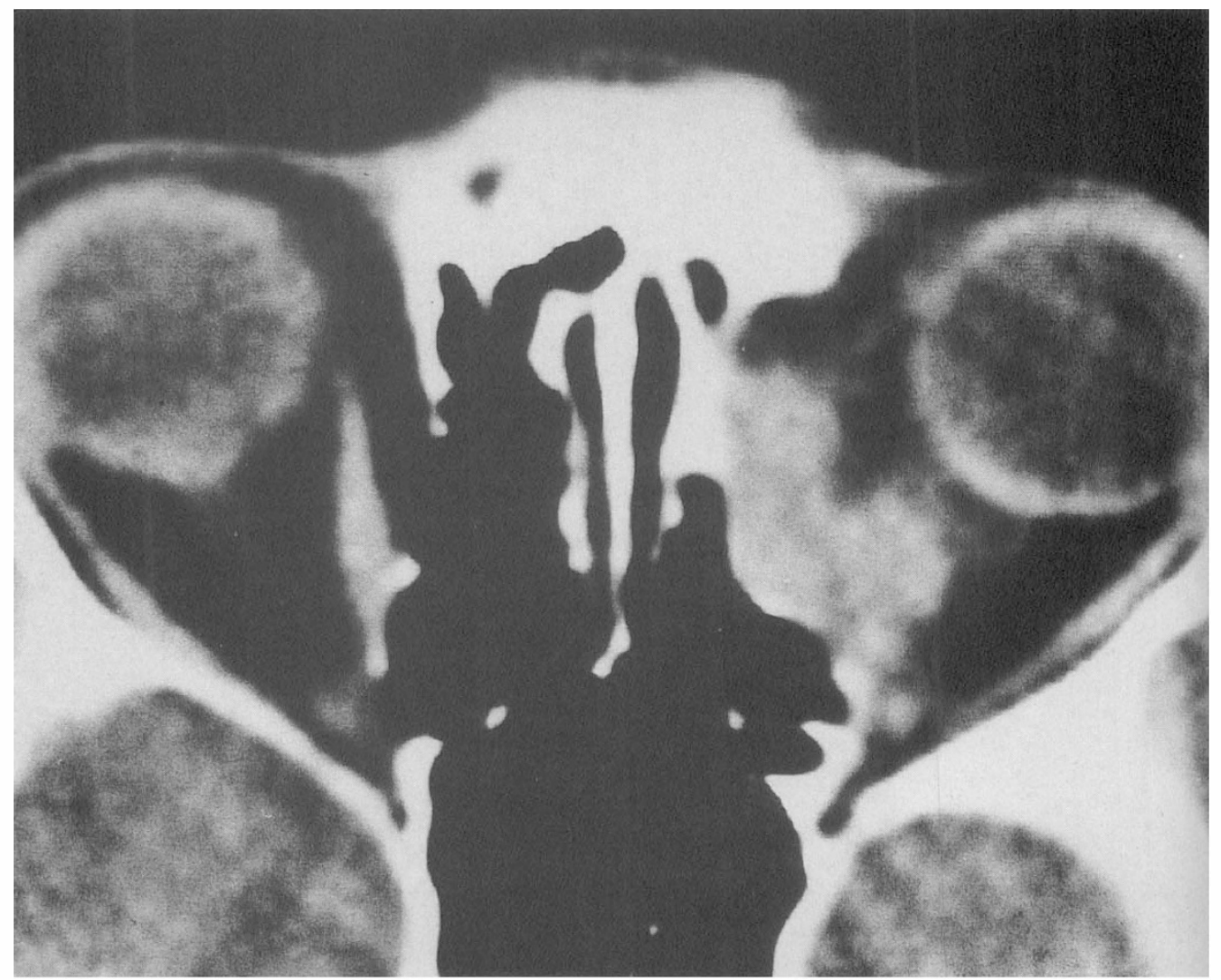

Fig. 15. Case 2: Postoperative CAT scan demonstrating residual tumour prolapsing into medial orbital defect with resolution of protosis. 


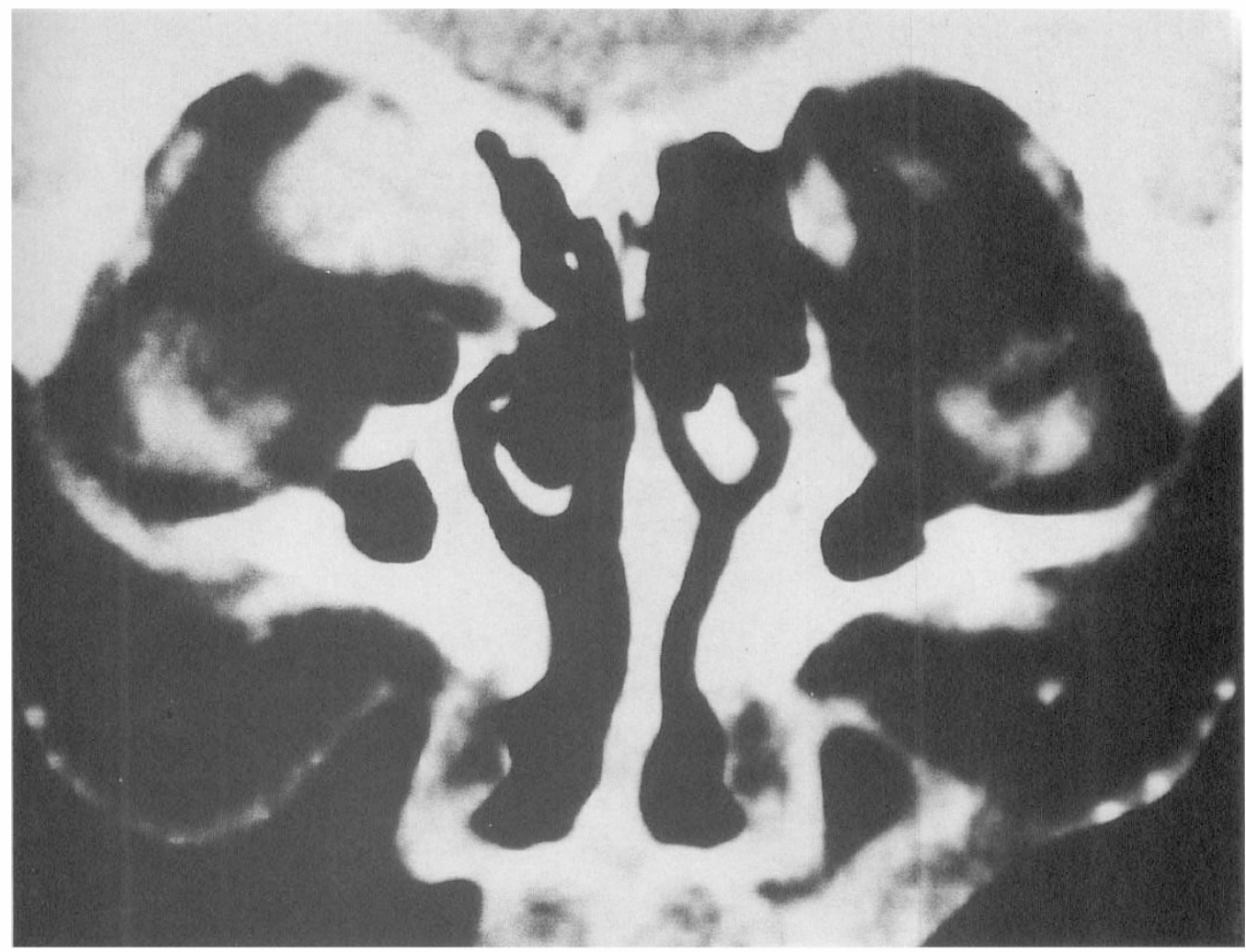

Fig. 16. Case 2: Postoperative CAT scan: Coronal view.

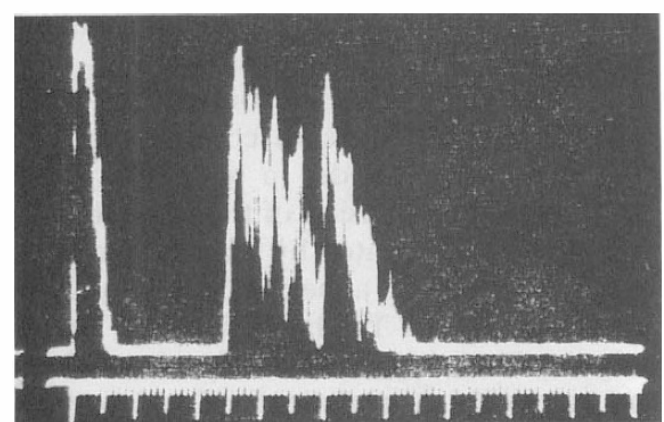

Fig. 17. Case 3: A-mode ultrasonogram of cavernous haemangioma demonstrating moderate internal reflectivity.

to play in the diagnosis of this tumour, which usually has a stagnant circulation and no prominent arterial supply. The information derived from their use adds little to that derived from the less invasive modalities of CAT scanning and ultrasonography.
It has been emphasised, however, that rare examples of well-delineated or encapsulated malignant intraconal lesions, e.g. haemangiopericytoma, do occur which CAT scanning and ultrasonography may mistake for orbital cavernous haemangioma. ${ }^{3}$

In their review of 66 patients with orbital cavernous haemangioma, Harris and Jakobiec found that $25 \%$ of the patients suffered a reduction in visual acuity as a result of surgery. ${ }^{4}$ All of the lesions in their series were managed by an anterior or lateral approach. They found that a higher percentage of the group with decreased vision postoperatively, in comparison with the entire series, had impalpable lesions situated deep in the orbit causing striae, disc swelling, envelopment of the optic nerve, or adhesion to the optic nerve, periosteum, or muscles. They also point out the significant incidence of complications secondary to orbital surgery per se. They conclude that the patient who 


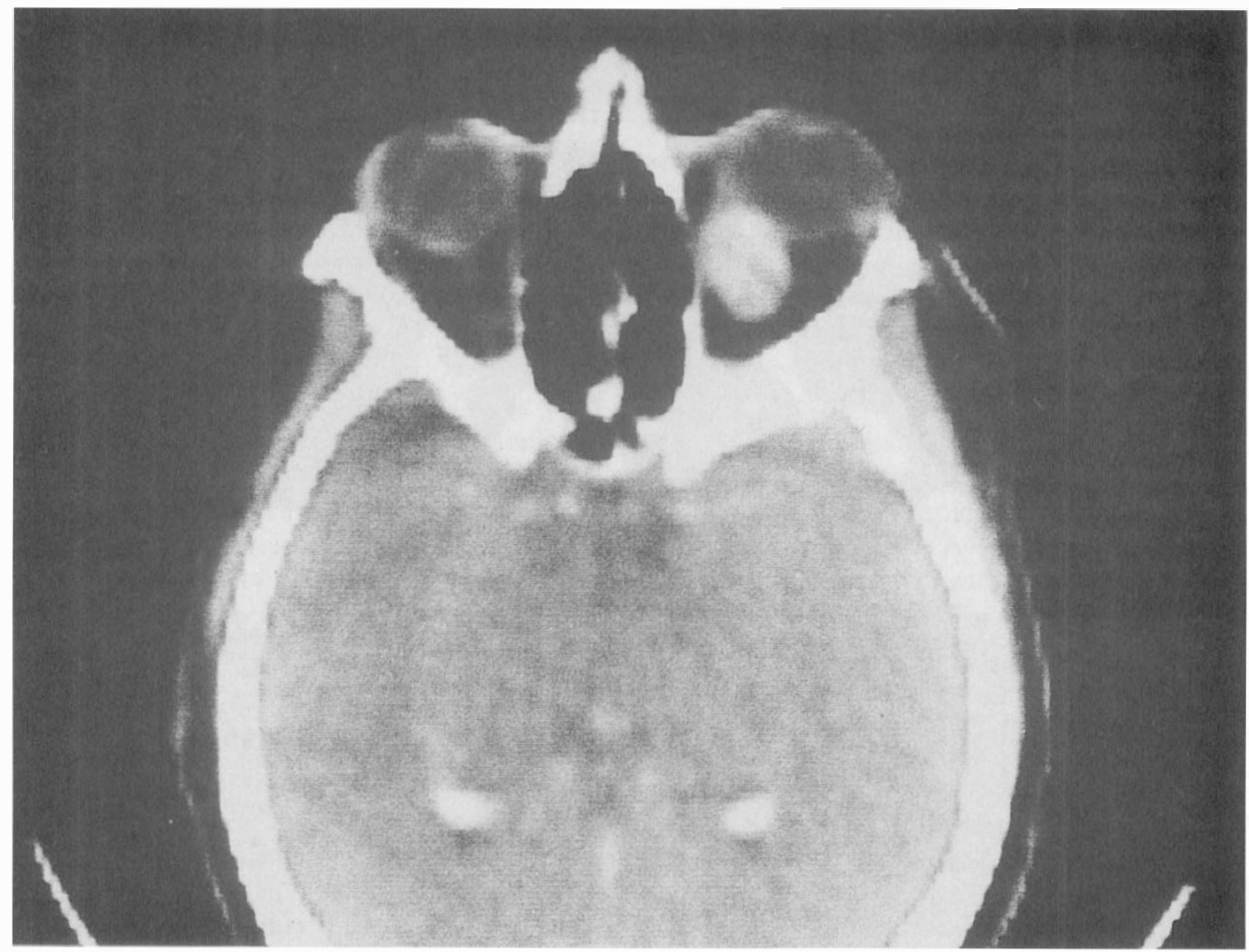

Fig. 18. Case 3: Preoperative CAT scan demonstrating orbital cavernous haemangioma and proptosis.

may benefit most from surgery is the one with true visual loss secondary to optic nerve compression, but point out that the location of the lesion in such a case makes uncomplicated delivery more difficult.

Wright maintains that orbital cavernous haemangiomas should be removed as soon as the diagnosis has been made to prevent damage to the orbital structures unless the patient is elderly or in poor general health. ${ }^{7} \mathrm{He}$ advocates a lateral orbitotomy to approach this lesion in the intraconal space, with removal of the tumour by meticulous dissection. Nevertheless, he reports one case of blindness from damage to the posterior ciliary arteries following such a procedure. ${ }^{7}$

We feel that medial orbitotomy combined with medial orbital decompression permits a safe and adequate access to the retrobulbar intraconal space for the management of medially-placed orbital cavernous haemangiomas. If the tumour cannot be removed easily and safely, we feel that it is justified merely to debulk it and leave it in situ.

The medial orbital decompression then relieves both optic nerve compression and proptosis and provides a space for slow asymptomatic expansion of the tumour. This effect is well illustrated by Case 2 . The postoperative cosmetic appearance is very satisfactory (Fig. 12). To the best of our knowledge, orbital decompression performed for the surgical management of an orbital haemangioma has only been described once before ${ }^{8}$ but in this case the diagnosis was not confirmed histopathologically. It will be interesting to report on the long-term followup of case 2 .

\section{References}

${ }^{1}$ Reese AB: Tumours of the eye. 3rd ed. Hagerstown: Harper \& Row. 1976; 264.

2 Wright JE: Orbital vascular anomalies. Trans 
Am Acad Ophthalmol Otolaryngol. 1974; 78: 606-16.

${ }^{3}$ Ruchman MC, and Flanagan J: Cavernous hemangiomas of the orbit. Ophthalmology 1983; 90: 1328-36.

${ }^{4}$ Harris GJ, and Jakobiec FA: Cavernous hemangioma of the orbit. J. Neurosurg. 1979; 51: 219-28.

${ }^{5}$ Davis RD, Hesselink JR, Dallow RL, Grove AS: CT and ultrasound in the diagnosis of cavernous hemangioma and lymphangioma of the orbit. J-Comput-Tomogr. 1980; 4: 98-104.
${ }^{6}$ Coleman DJ, Jack RL, Franzen LA: High resolution B-scan ultrasonography of the orbit. II. Hemangiomas of the orbit. Arch Ophthalmol. 1972; 88: 368-74.

${ }^{7}$ Wright JE: Doyne lecture: Current concepts in orbital disease. Eye, 1988; 2: 1-11.

${ }^{8}$ Rowbotham GF, Little E: Hemangioma of the orbit. Case causing periodic obstruction and exophthalmos treated successfully by a posteriorly placed orbital decompression. $\mathrm{Br} J$ Ophthalmol 1966; 50: 47-9. 